\title{
Surgical Revascularization of Lower Limb for Chronic Occlusive Arterial Diseases-Outcome of 35 Patients
}

\author{
REZWANUL HOQUE ${ }^{1}$, MD. ASLAM HOSSAIN ${ }^{1}$, SABRINA SHARMEEN HUSAIN ${ }^{1}$, MAHMUDUR RAHMAN \\ KHANDOKER $^{2}$, MOSTAFANURUZZAMAN ${ }^{3}$, MD.ALAUDDIN ${ }^{1}$, MD.ANWARUL ISLAM ${ }^{1}$
}

${ }^{1}$ Department of Cardiac Surgery, Bangabandhu Sheikh Mujib Medical University(BSMMU), ${ }^{2}$ Department of Anesthesiology, Dhaka Medical College, Dhaka, ${ }^{3}$ Department of Anesthesiology, Bangabandhu Sheikh Mujib Medical University (BSMMU), Dhaka

Address for correspondence: Dr. Md. Rezwanul Hoque (Bulbul), Associate Professor, Department of Cardiac Surgery, Bangabandhu Sheikh Mujib Medical University (BSMMU), Bangladesh, E-mail drrhbulbul @yahoo.com

\begin{abstract}
Background: Chronic lower limb ischemia is a dreadful disease and may present with intermittent claudication, rest pain and ischemic gangrene. Apart from life style modification and treating risk factors either angioplasty and stenting or surgical bypass are the mainstay of treatment. For infrainguinal bypasses reversed saphenous venous grafts are the conduits of choice because it is autologous, and have good patency rate. Endarterectomy is used in vessels of large caliber and may be added to bypass procedure.

Objective: To study the outcome of surgical revascularization of the lower limb for chronic ischemia using reversed saphenous venous grafts.

Methods: Thirty five cases of lower limb bypass surgery using reversed saphenous vein grafts were done for critical chronic lower limb ischemia from January, 2004 to December, 2008 and were analyzed for clinical success. The clinical success was defined as freedom from symptoms, avoidance of further revascularization, surgical or interventional or freedom from further amputation. The bypass procedures were femoro-popliteal, femoro-distal, femoral endarterectomy plus bypass, profundoplasty plus bypass. Data were collected, analyzed and results were recorded before discharge from the hospital, at 3 months, at 6 months and thereafter yearly follow up for up to 3 years.

Results: The age range was 55 to 72 years (mean $62.34 \pm 05.98$ SD), 30 cases were male, 5 cases were female, all male patients were chronic smokers, 28 cases were diabetic, and 26 cases were hypertensive. Altogether 48 procedures were done, femoro-popliteal bypass were done in 30 cases, femoro-distal bypass were done in 7 cases (distal anastomotic sites were anterior tibial, posterior tibial or arteria dorsalis pedis), 9 cases had common femoral endarterectomy after which femoro-popliteal bypass were done, profundoplasty with femoro-popliteal bypass were done in 2 cases, 10 patient had to undergo either toe or transmetatarsal amputation. Three cases were subjected to below knee amputation at 2 to 3 years follow up due to recurrent ischemic rest pain with patchy gangrene. These were patients with femoral endarterectomy cases. The patency rate of grafts at 3 years was $65 \%$ for femoro-popliteal, $60 \%$ for femoro-distal, $57 \%$ for femoral endarterectomy with bypass and $58 \%$ for profundoplasty with bypass procedure.

Conclusion: The graft patency rate and limb salvage rate for infrainguinal bypass procedure using reversed saphenous vein graft were quite satisfactory. Cessation of smoking, anti platelet and lipid lowering drug therapy, daily brisk walking for one hour and lifestyle modification improved the claudication distance and saved the limb and life in the study population.
\end{abstract}

Introduction

Majority of the cases of chronic limb ischemia are caused by insidious and relentless progress of atheromatous plaque buildup, and, terminally by thrombotic occlusion of remaining lumen. The risk factors associated with the development of atherosclerosis such as cigarette smoking, diabetes mellitus, hypertension, dyslipidemia, hyperhomocysteinemia entail 2 to 6 fold incremental risk of development of chronic occlusive arterial disease and 3 to 10 fold increased risk of development of intermittent claudication. ${ }^{1}$ The prevalence rate of this disease varies depending on the diagnostic criteria such as Rose questionnaire for intermittent claudication, pulse examination, ankle-brachial pressure index (ABI), pulsewave velocity index. The ankle-brachial index though overestimated in diabetes and chronic renal failure due to 
calcific arterial wall, is normally $>1.0$; the index being decreased to 0.50 to 0.90 in patients with claudication and to lower levels in patients with pain at rest or tissue loss. When the objective noninvasive ABI and pulse-wave velocity techniques were used, the prevalence of lower extremity peripheral arterial disease is $2.5 \%$ among individuals 60 years and younger, $8.3 \%$ among those aged 60 to 69 years, and $18.8 \%$ among those 70 years and older. ${ }^{2}$ Other non invasive vascular laboratory tests include segmental limb systolic pressure measurement, toe pressure and toe brachial pressure index and pulse volume recording. In segmental limb systolic pressure measurement a sphygmomanometer cuff is placed at a given level with a Doppler probe over one of the pedal arteries, and the systolic pressure in the major arteries under the cuff is measured. The location of occlusive lesions is apparent from the pressure gradients between the different cuffs, a gradient of $20 \mathrm{~mm}$ Hg or more between adjacent segments indicates arterial stenosis in the intervening segment. ${ }^{1,3}$ For patients with suspected lower extremity peripheral arterial disease the pulse volume recording may be an initial noninvasive screening test and it can also predict critical limb ischemia and risk of amputation.

The pulse volume recording technique is useful as an initial diagnostic test for patients with suspected lower extremity peripheral arterial disease and to assess limb perfusion after revascularization procedures, and it can predict risk of critical limb ischemia and amputation. ${ }^{1}$

In patients with diabetes mellitus or chronic renal failure the vessels are non compressible sometimes calcify having highly elevated ankle pressure $(\geq 250 \mathrm{mmHg}$ ) or ankle brachial index $>1.40$. Measurement of toe pressure which is normally approximately $30 \mathrm{mmHg}$ less than ankle pressure, using special occlusion cuff on the first or second toe with a flow sensor may be useful in these cases. An abnormal toe brachial index is $<0.70^{1}$

The imaging studies include angiography, color-assisted duplex ultrasonography, magnetic resonance angiography (MRA) and computed tomography angiography (CTA). Angiography having its own drawback is still considered as the gold standard for evaluating peripheral arterial disease (PAD), and is the most readily available and widely used imaging technique. Color-assisted duplex ultrasonography can localize the lesion accurately and is useful in delineating between stenotic and occlusive lesions. The main advantage of MRA particularly gadolinium contrast based MRA (CE-MRA) is its ability to provide rapid high-resolution three- dimensional (3D) imaging of the entire abdomen, pelvis and lower extremities in one setting. Multislice MDCTA enables fast imaging of the lower extremity and abdomen in a single breath-hold at submillimeter isotropic voxel resolution. ${ }^{4}$

The severity of chronic lower limb ischemia has been graded, Fontaine stage I to IV and Rutherford category 1 to $6 .{ }^{5}$ The risk of limb loss with intermittent claudication alone is around $2 \%$ at 5 years whereas the risk of major amputation increases dramatically when symptoms of rest pain or tissue loss appears. ${ }^{1}$ Coronary atherosclerosis is a common accompaniment and some $28 \%$ of patient has severe triple vessel disease. ${ }^{1}$ The extent and severity of the disease and the risk factor involved also vary so a detailed work up is undertaken before rendering therapeutic manipulation. The management of patients with lowerextremity peripheral arterial disease is two-pronged: first, the risk factors important in the progression of generalized atherosclerosis are addressed followed by interventions such as pharmacotherapy and endovascular therapy or surgery to relieve the lower-extremity symptoms. The risk factor modification includes complete abstinence of smoking, control of diabetes mellitus, control of hypertension, correction of dyslipidaemia, daily physical exercise including walking and regaining ideal body weight. The surgical therapies for peripheral arterial diseases rely on two modalities of treatment, endarterectomy and bypass procedure. Endarterectomy is suitable for localized disease in larger caliber vessel such as abdominal aorta, common iliac arteries; the long term patency rate in smaller vessels is far more inferior to bypass procedure. For aortoiliac diseases synthetic grafts are conduits of choice, for infrainguinal procedures autologus venous grafts either reversed or in situ give better result, although prosthetic graft may be used if they do not cross knee joint.

\section{Materials and Methods:}

Thirty five cases lower limb bypass surgery were done for critical chronic lower limb ischemia from January 2004 to December 2008 and were analyzed for clinical success. Patients were followed up for at least 3 years. The clinical success was defined as freedom from symptoms, avoidance of further revascularization (Surgical or interventional) or freedom from further amputation. In all cases reversed saphenous venous grafts were used as 
conduits and the bypass procedures involved were femoro-popliteal, femoro-distal, femoral endarterectomy plus bypass, profundoplasty plus bypass. Elaborate history taking and thorough physical examination were done and demographic data including age and sex, risk factors and history of previous vascular operation were recorded. In addition to routine investigation, all patients were assessed by duplex scan of the lower limb vessels, peripheral contrast angiography before operation and by clinical examination of pulses, duplex scan and/ or computerized tomographic angiography (CTA) at follow up period. Data were collected, analyzed and results were recorded before discharge from the hospital, at 3 months, 6 months and thereafter at yearly follow up.

\section{Results:}

The age range was 55 to 72 years (Mean $62.34 \pm 05.98$ SD), 30 cases were male, 5 cases were female, all male patients were chronic smokers, 28 cases were diabetic, and 26 cases were hypertensive. All patients were having rest pain with 10 cases having gangrenous changes in the toes, 5 patients had ischemic patchy gangrene in the leg with edematous legs. Thirteen patients had bilateral involvement in which staged operations were performed.

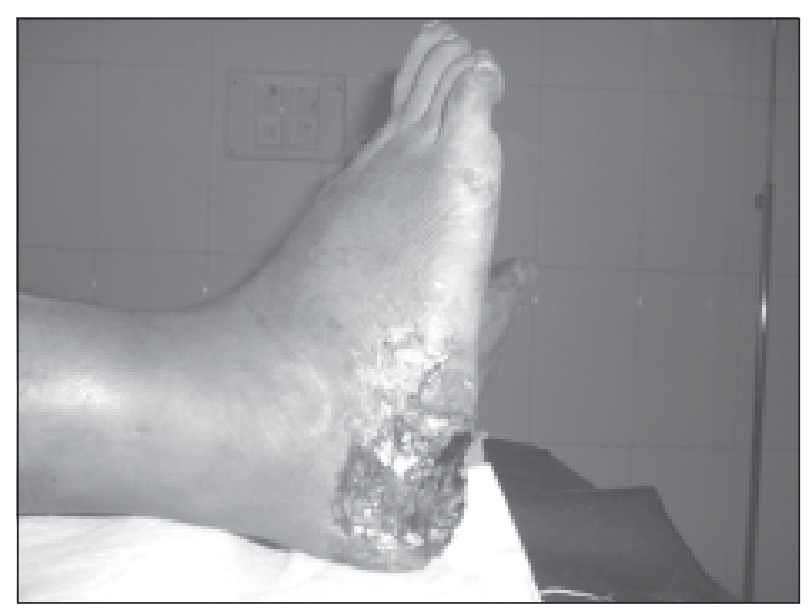

Fig.-1: Showing ischemic patchy gangrene in the heel area.

All operations were done under epidural anaesthesia and postoperative pain relief were ensured by intermittent bolus epidural analgesia supplemented by non-steroidal anti inflammatory drug and narcotic analgesia as needed. Altogether 48 procedures were done, femoro-popliteal bypass were done in 30 cases, femoro-distal bypass were done in 7 cases (distal anastomotic sites were anterior tibial, posterior tibial or arteria dorsalis pedis), 9 cases had common femoral endarterectomy after which femoropopliteal bypass were done, profundoplasty with femoropopliteal bypass were done in 2 cases, 10 patient had to undergo either toe or transmetatarsal amputation , 5 cases needed excision of the gangrenous skin only.

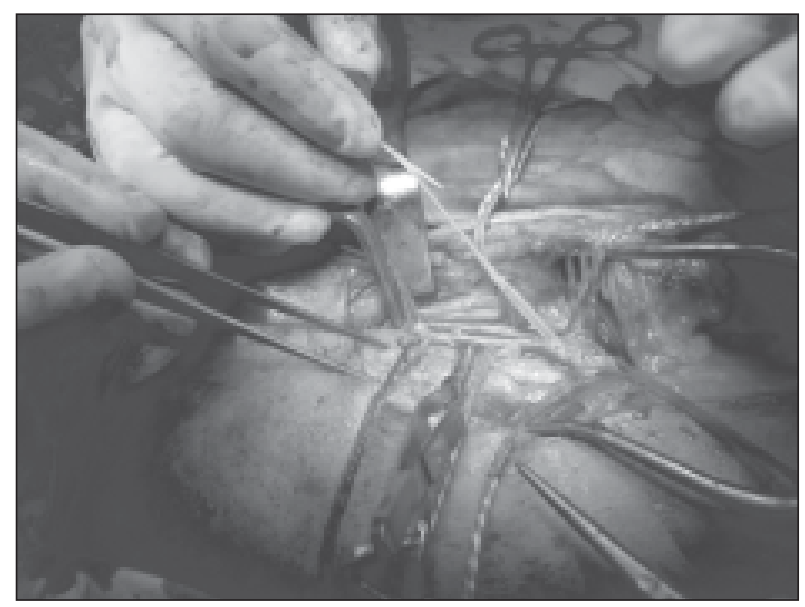

Fig.-2: Showing common femoral endarterectomy with venous patch angioplasty.

Three cases were subjected to below knee amputation at 2 to 3 years follow up due to recurrent ischemic rest pain with patchy gangrene. These were patients with femoral endarterectomy cases. The patency rate of grafts at 3 years was $65 \%$ for femoro-popliteal, $60 \%$ for femoro-distal, $57 \%$ for femoral endarterectomy with bypass and $58 \%$ for profundoplasty with bypass procedure. Limb losses due to occlusion of the graft are less due to development of collateral circulation on antiplatelet therapy, daily walking and lifestyle modification.

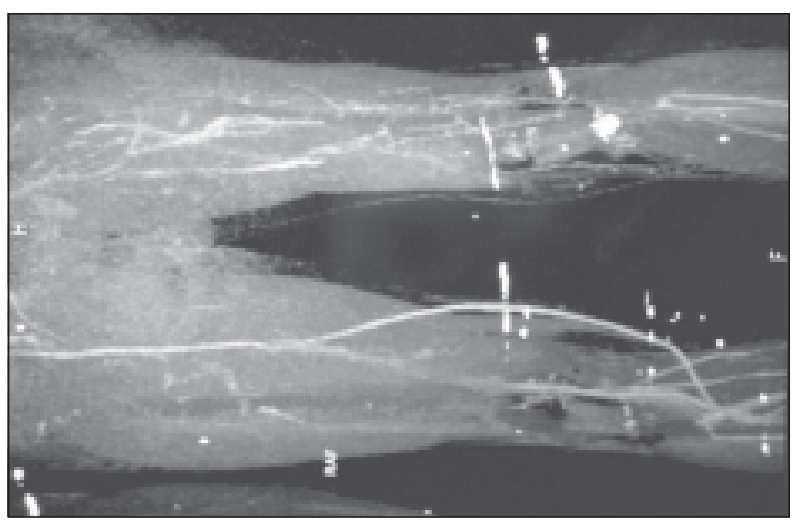

Fig.-3: CT angiogram showing patent Femoro-popliteal bypass raft at 3 years, the patient needed PTFE Iliofemoral bypass graft in the opposite limb. 


\begin{tabular}{lccc}
\hline Procedure & $\begin{array}{c}\text { Number } \\
\text { (Percentage) }\end{array}$ & $\begin{array}{c}\text { Graft patency } \\
\text { rate at 3 years }\end{array}$ & $\begin{array}{c}\text { Outcome } \\
\text { at 3 years }\end{array}$ \\
\hline Femoro-popliteal bypass & 30 & $65 \%$ & satisfactory \\
Femoro-distal bypass & 9 & $60 \%$ & satisfactory \\
Femoral endarterectomy plus bypass & 7 & $57 \%$ & 3 cases required below \\
knee amputation \\
Profundoplasty plus bypass
\end{tabular}

\section{Discussion}

The long term patency rate of percutaneous transluminal angioplasty with stenting is inferior to open surgical bypass procedure particularly in crural vessels (approximating $70 \%$ to $80 \%$ at 5 years). The 3 - year patency rate of aortoiliac angioplasty with stenting is around 86 percent whereas for infrainguinal bypass it is around 60 percent and considering the dismal prognosis of angioplasty procedure for crural vessels bypass procedure should be considered as the first line of therapy. ${ }^{6,7}$

Desai TR et al (2001) reported a retrospective analysis of infrainguinal bypass procedure (274 patients, 351 procedures) over a period of 5 years. The procedures involved in anastomosing the distal end of the graft to a vessel with at least one good run off and to a blind vessel with collateral flow only. The graft patency rate were similar in both groups (67 $\pm 7 \%$ vs. $64 \pm 4 \%$ ), p not significant) although the limb loss rate was significantly higher in the blind outflow group. ${ }^{8}$

A multicenter randomized prospective study revealed that interposition vein cuff improved the short-term and medium-term patency and limb salvage rates of femoralbelow-knee popliteal artery polytetrafluoroethylene (PTFE) bypass procedures compared to femoral-above knee bypass procedures. ${ }^{9}$

Chronic smoking, diabetes mellitus and hypertension were major risk factors in the population under present study and were in an advanced stage of the disease. The graft patency rate was quite comparable and limb salvage rate were quite satisfactory. Only 3 cases needed below knee amputation. Cessation of smoking, ant platelet and lipid lowering drug therapy, daily brisk walking for one hour and lifestyle modification improved the claudication distance and saved the limb and life in the study population.

HMG-CoA reductase inhibitors (statins) broadly reduce cardiovascular events, effects that are only partly related to cholesterol lowering. Recent studies suggest important anti-inflammatory and antiproliferative properties of these drugs. A retrospective analysis on the infrainguinal autologus venous bypass procedures showed improved result after statin therapy. Patients on statins had higher primary-revised ( $94 \% \pm 2 \%$ vs. $83 \% \pm-5 \%$; $\mathrm{P}<.02)$ and secondary $(97 \%+2 \%$ vs. $87 \% \pm 4 \%$; $\mathrm{P}<.02)$ graft patency rates at 2 years. The risk of graft failure was 3.2-fold higher (95\% confidence interval, 1.04-10.04) for the control group not taking statin. ${ }^{10}$

\section{References}

1. Hirsch AT, Haskal ZJ, Hertzer NR, Bakal CW, Creager MA, Halperin JL et al. Peripheral Arterial Disease: ACC/AHA 2005 guidelines for the management of patients with peripheral arterial disease (lower extremity, renal, mesenteric, and abdominal aortic): J Am Coll Cardiol 2006;47:1239-312.

2. Kannel WB. The demographics of claudication and the aging of the American population. Vasc Med 1996; 1:60-4.

3. Begelman SM, Jaff MR. Noninvasive diagnostic strategies for peripheral arterial disease. Cleve Clin J Med 2006; 73(Suppl 4): S22-29.

4. Norgren L, Hiatt WR, Dormandy JA, Nehler MR, Harris KA, Fowkes FG. TASC II working group. Inter-Society consensus for the management of peripheral arterial disease (TASC II). Eur J Vasc Endovasc Surg 2007; 33(Suppl 1): S1-75.

5. Dormandy JA, Rutherford RB. Management of peripheral arterial disease (PAD). TASC Working Group. Transatlantic Inter-Society Consensus (TASC). J Vasc Surg 2000; 31(1 pt 2):S1-S296.

6. Taylor LM Jar, Edwards JM, Porter JM. Present status of reversed vein bypass grafting: five-year results of modern series. J Vasc Surg.1990; 11:193-206.

7. Belkin M, Knox J, Donaldson MC, Mannick JA, Whittemore AD. Infrainguinal arterial reconstruction with nonreversed greater saphenous vein. J VascSurg. 1996; 24:957-62.

8. Desai TR, Meyerson SL, Skelly CL, MacKenzie KS, Bassiouny HS, Katz D, et al. Patency and limb salvage after infrainguinal bypass with severely compromised (〉’blind〉”) outflow. Arch Surg. 2001 Jun;136(6):635-42

9. Stonebridge PA, Prescott RJ, Ruckley CV. Randomized trial comparing infrainguinal polytetrafluoroethylene bypass grafting with and without vein interposition cuff at the distal anastomosis, J Vasc Surg 1997;26:543-50.

10. Abbruzzese TA, Havens J, Belkin M, Donaldson MC, Whittemore AD, Liao JK et al. Statin therapy is associated with improved patency of autogenous infrainguinal bypass grafts. J Vasc Surg. 2004 Jun; 39(6):1178-85.

11. Singh D. Management of Peripheral Arterial Disease: An Overview. The Internet Journal of Surgery. 2006 Vol 8 No 1 available at. http://www.ispub.com/journal/the_internet _journal_of_surgery/volume_8_number_1/article/ management_of_peripheral_arterial_disease_an_overview.html 